https://doi.org/10.48009/1_iis_2010_90-99

\title{
USING OBJECT-RELATIONAL DATABASE TECHNOLOGY TO SOLVE PROBLEMS IN DATABASE DEVELOPMENT
}

Ming Wang, California State University, ming.wang @ calstatela.edu

\begin{abstract}
The emergence of object-relational database (ORDB) technology into the commercial database market has caused the database professional's attention in seeking how to utilize its object-oriented features in the database development. Although the ORDB technology is already available for use in all the major DBMS products, its industrial adoption rate is relatively low. The purpose of the paper is to promote utilization of ORDBMS features in for industrial database application practitioners and provide them the solutions to the problems in their database development. The goal of the paper is to present how to use the object-relational database management system (ORDBMS) to overcome relational database $(R D B)$ existing problems and improve database performance in the database development using features of ORDB technology. The paper introduces how to use the specific ORDB technology to solve normalization problems in 1) Transitive dependency, 2) Multi-value attributes, and 3) Non-1st Normal Form and also provides the solutions to data complexity problems with specific ORDBMS techniques: 1) object view, 2) object inheritance, and 3) object and integration. The paper concludes with a summary and discussion of significance and advantages of using ORDBMSs to solve the problems in the database development. The author utilizes Oracle as a tool to demonstrate how to overcome some weaknesses of relational DBMS. The ORDBMS script in the study has been tested in the Oracle 9i, $10 \mathrm{~g}$, and $11 \mathrm{~g}$ environment.
\end{abstract}

Keywords: Object-relational database, Database Curriculum, Oracle Database, Normalization

\section{INTRODUCTION}

Object-relational database technology has emerged as a way of enhancing object-oriented features in relational database management systems (RDBMSs).
ORDBMS enhances object-oriented technology into the relational database management system (RDBMS) and extends traditional RDBMS with object-oriented features [4]. As an evolutionary technology, ORDBMS allows users to take advantages of reuse features in object-oriented technology, to map objects into relations and to maintain a consistent data structure in the existing RDBMS.

The success of relational DBMSs cannot be denied, but they experience difficulty when confronted with the kinds of "complex data" found in advanced application areas such as hardware and software design, science and medicine, and mechanical and electrical engineering. To meet the challenges, Oracle, IBM and Microsoft have moved to incorporate object-oriented database features into their relational DBMSs under the name of objectrelational DBMSs. The major database vendors presently support object-relational data model, a data model that combines features of the object-oriented model and relational model [17]. Although the ORDB technology is already available for use in all the major DBMS products, its industrial adoption rate is not very high. One of the major criticisms of ORDBMS is that its complexity results in the loss of the essential simplicity and purity of the relational database model. It is challenging to for industrial application developers who have traditional relational database background to adopt the emergent ORDB technology.

The purpose of the paper is to promote utilization of ORDBMS features in for industrial database application practitioners and provide them the solutions to the problems in their database development. The paper first introduces the background and features of ORDBMS, then presents how to use the specific ORDBMS techniques to solve normalization problems in 1) Transitive dependency, 2) Multi-value attributes, 3) and Non-1st Normal Form, and how to use the specific ORDBMS 
features: 1) object view 2) object inheritance and 3) object integration to solve data complexity problems. Finally, the paper concludes with a summary and discussion of using ORDBMSs as remedy to solve the relational database problems. Many of the ORDBMS features appear in Oracle. Thus, the author utilizes Oracle as a tool to demonstrate how to overcome some weaknesses of relational DBMS. The ORDBMS script in the case study has been tested in the Oracle 9i, 10g, and 11g SQLPlus environment.

\section{OBJECT-RELATIONAL DB TECHNOLOGY}

The object-relational database technology occurrence can be traced back to the middle of 1990s after emergence of object-oriented database (OODB). In their book "Object-relational DBMSs: the Next Great Wave", Stonebraker and Moore [18] define their four-quadrant view (two by two matrix) of the data processing world: relational database, objectrelational database, data file processing, and objectoriented database. Their purpose is to indicate the kinds of problems each of four-quadrants solves. As will be seen, "one size does not fit all"; i.e. there is no DBMS that solves all the applications. They suggest that there is a natural choice of data manager for each of the four database applications. They conclude why the problems addressed by object-relational DBMSs are expected to become increasingly important over the next decade. As such, it is "the next wave".

Theoretically, as Stonebraker and Moore [18] predict in their four-quadrant view of the database world, ORDBMS has been the most appropriate DBMS that processes complex data and complex queries. The object-oriented database management systems have made limited inroads during the 1990's, but have since dying off. Instead of a migration from relational to object-oriented systems, as was widely predicted around 1990, the vendors of relational systems have incorporated many object-oriented database features into their DBMS products. As a result, many DBMS products that used to be called "relational" are now called "object-relational" [7].

Practically, ORDBMS bridges the gap between OODBMS and RDBMS by allowing users to take advantage of OODB'MSs great productivity and complex data type without losing their existing investment in relational data [3]. In fact, an ORDBMS engine supports both relational and object- relational features in an integrated fashion [6]. The u0nderlying ORDB data model is relational because object data is stored in tables or columns. ORDB designers can work with familiar tabular structures and data definition languages (DDLs) while assimilating new object-oriented features [10].It is essentially a relational data model with objectoriented extensions. In response to the evolutional change of ORDB technology, SQL:1999 started supporting object-relational data modeling features in database management standardization and SQL:2003 continues this evolution [5]. Currently, all the major database vendors have upgraded their relational database products to object-relational database management systems to reflect the new SQL standards [9] and ready to be used by industrial practitioners.

Although each of object-relational DBMS vendors has implemented OO principles: encapsulation and inheritance in their own way, all of them share the combination of the $\mathrm{OO}$ principles and follow SQL standardization, incorporate object-oriented paradigms. All the ORDBMSs have the ability to store object data and methods in databases. Many of SQL:2003 standard ORDBMS features appear in Oracle. They are listed as follows.

Object Types: User-defined data types (UDT) or abstract types (ADT) are referred to as object types.

Functions/Methods: For each object type, the user can define the methods for data access. Methods define the behavior of data.

Varray: The varray is a collection type that allows the user to embed homogenous data into an array to form an object in a pre-defined array data type.

Nested table: A nested table is a collection type that can be stored within another table. With a nested table, a collection of multiple columns from one table can be placed into a single column in another table.

Inheritance: With Object type inheritance, users can build subtypes in hierarchies of database types in ORDBs.

Object View: Object view allows users to develop object structures in existing relational tables. It allows data to be accessed or viewed in an object- 
oriented way even if the data are really stored in a traditional relational format.

\section{ORDBMS FOR NORMALIZATION}

Normalization is a logical data modeling technique for the development of a well structured relational database. The process is decomposing tables with anomalies to produce smaller tables. Traditional normalization processes are normalizing tables in non-1NF form and multi-value attributes to at least $3 \mathrm{NF}$; and removing transitive dependency. Such processes can be eliminated if ORDB technology is used.

\section{Object Type \& Transitive Dependency}

The address attribute is usually split into four columns such as street, city, state and zip code in order to store address dada in a customer table since it is a composite attribute in a traditional database.

Customer table

\begin{tabular}{|l|l|l|l|l|l|l|}
\hline $\begin{array}{l}\text { Cu_i } \\
\mathrm{d}\end{array}$ & $\begin{array}{l}\text { Firs } \\
\mathrm{t}\end{array}$ & $\begin{array}{l}\text { Las } \\
\mathrm{t}\end{array}$ & Street & City & $\begin{array}{l}\text { Stat } \\
\mathrm{e}\end{array}$ & Zip \\
\hline 1 & $\begin{array}{l}\text { Joh } \\
\mathrm{n}\end{array}$ & $\begin{array}{l}\text { Smi } \\
\mathrm{h}\end{array}$ & $\begin{array}{l}12 \\
\text { Pine }\end{array}$ & Bell & CA & $\begin{array}{l}9020 \\
1\end{array}$ \\
\hline 2 & $\begin{array}{l}\text { Ma } \\
\text { ry }\end{array}$ & Fox & $\begin{array}{l}6 \\
\text { Circle }\end{array}$ & Brea & CA & $\begin{array}{l}9282 \\
1\end{array}$ \\
\hline
\end{tabular}

The above Customer table is in Second Normalization Form (2NF) and violates the Third Normalization Form (3NF) rule because there is the transitive dependency in the customer table. Zip is a determinant of street, city and state. Functional dependency analysis shows transitive dependency:

Zip -> Street, City, State (transitive dependency)

There are three solutions to this transitive dependency problem. Solution 1 keeps the customer table in the Second Normalization Form (2NF) though it is not an ideal normal form for a relational database.

Solution 2 is to create a new customer address table by splitting the address from the original customer table (3NF). This solution implies more joins of records in the Customer table and Zip table.
Customer Table

\begin{tabular}{|l|l|l|l|}
\hline Cu_id & First & Last & Zip \\
\hline 1 & John & Smith & 90201 \\
\hline 2 & Mary & Fox & 92821 \\
\hline
\end{tabular}

Zip Table

\begin{tabular}{|l|l|l|l|}
\hline Zip & Street & City & State \\
\hline 96123 & 12 Pine & Bell & CA \\
\hline 25678 & 6 Circle & Brea & VA \\
\hline
\end{tabular}

Solution 3 is to store all the customer address information in one column. This solution creates difficulty in data retrieval. For example, it is impossible to retrieve or sort customer records by city, state or zip code.

Customer Table

\begin{tabular}{|l|l|l|l|}
\hline Cu_id & First & Last & Address \\
\hline 1 & John & Smith & 12 Pine, Bell, CA 90201 \\
\hline 2 & $\begin{array}{l}\text { Mar } \\
\text { y }\end{array}$ & Fox & 6 Circle, Brea, CA 92821 \\
\hline
\end{tabular}

None of the above three solutions is considered ideal in terms of efficient database design and operations. The first solution is not satisfactory since $2 \mathrm{NF}$ is not ideal for relational database design. The second solution implies that more joins might occur in the query process, since the zip table has been added to the database. The third solution creates difficulty in data retrieval. For example, it is impossible to retrieve or sort customer records by city, state, or zip code.

With ORDBMS technology, the attribute address can be defined as a user-defined abstract data type with a number of attributes using s the same internal format. User-defined types (UDT) or abstract data types (ADT) are referred to as object types. Object types are used to define either object columns or object tables. The following UML Customer class illustrates the address object column.

\begin{tabular}{|l|}
\hline \multicolumn{1}{|c|}{ Customer } \\
\hline$-<$ PK> cust_id : Integer \\
-name : Object \\
-address : Object \\
-<multivalued $>$ phone : Object \\
\hline +getFullName() \\
\hline
\end{tabular}


Object types need to be defined before the custome table. The following SQL statements define the object types: address_ty and name_ty.

$\begin{array}{ll}\text { CREATE } & \text { OR REPLACE TYPE address_ty AS } \\ \text { OBJECT } & \\ \text { (street } & \text { NVARCHAR2(30), } \\ \text { city } & \text { VARCHAR2(25), } \\ \text { state } & \text { CHAR(2), } \\ \text { zip } & \text { NUMBER(10)); }\end{array}$

CREATE OR REPLACE TYPE name_ty AS OBJECT (

f_name VARCHAR2(25),

1_name VARCHAR2(25));

Mapping the above customer class, the following statement is used to create the Customer table with the CustName and CustAddress object columns using name_ty and address_ty. The column phone is to be added to the table later.

\section{CREATE TABLE Customer2( \\ Cust_ID Number(5), \\ CustName name_ty, \\ CustAddress address_ty);}

Object type constructors are used to insert object data into the table. The following INSERT statement uses constructors name_ty() and address_ty() to add data into the two object columns.

INSERT INTO Customer VALUES (1, name_ty ('John', 'Smith',), address_ty ('12 Road', 'Bell', 'CA', 90201));

The following statements retrieve the data from the Customer2 table.

SELECT c.custName.1_name, c.custAddress.City, c.custAddress.state FROM Customer2 c;

\begin{tabular}{|l|l|l|}
\hline CUSTNAME.L_NAME & CUSTADDRESS.CITY & CU \\
\hline John Smith & Bell & CA
\end{tabular}

SELECT * from Customer2;

$1 \mid$\begin{tabular}{l|l|}
$\begin{array}{l}\text { NAME_TY('John', } \\
\text { 'Smith') }\end{array}$ & $\begin{array}{l}\text { ADDRESS_TY('12 Pine', } \\
\text { 'Bell', 'CA', 90201) }\end{array}$ \\
\hline
\end{tabular}

\section{Varray and Multi-value Attributes}

In a relational model, multi-valued attributes are not allowed in the first normalization form. The traditional solution to the problem is that each multiple-valued attribute is handled by forming a new table in a relational database. If a table has five multivalued attributes, that table would have to be split into six tables. The Oracle ORDBMS allows users to create the varying length array (VARRAY) data type as a new data storage method for multi-valued attributes. The following statement defines a varray type of three VARCHAR2 string named varray_phone_ty to represent a list of phone numbers.

VARRAY is a collection type in ORDBMSs. A VARRAY consists of a set of objects that have the same predefined data type in an array. In a relational model, multi-valued attributes are not allowed in the first normalization form. The solution to the problem is that each multiple-valued attribute is handled by forming a new table. If a table has five multi-valued attributes, that table would have to be split into six tables after the First Form of normalization. To retrieve the data back from that original table, the student would have to do five joins across these six tables. ORDBMs allow multi-valued attributes to be represented in a database. ORDBMSs allow users to create the varying length array (VARRAY) data type can be used as a new data storage method for multivalued attributes. The following statement defines a VARRAY type of three VARCHAR2 strings named varray_phone_ty to represent a list of three phone numbers in the Customer2 table.

CREATE TYPE varray_phone_ty AS VARRAY(3) OF VARCHAR2(14);

ALTER TABLE Customer ADD (phones varray_phone_ty);

UPDATE customer

1211', SET phones $=($ varray_phone_ty('(800)555-

(800)555-1212','(800)555-1213'))

WHERE cust_id $=1$; 
INSERT INTO customer(phones) values (varray_phone_ty('(800)555-

1211',(800)555-1212',(800)555-1213'));

The above example shows that using the varying length array (VARRAY) data type not only can solve multi-value attribute problem for the customer table, but also can speed up the query process on customer data.

\section{Nested Table and Non-1NF}

A nested table is a table that can be stored within another table. With a nested table, a collection of multiple columns from one table can be placed into a single column in another table. Nested tables allow user to embed multi-valued attributes into a table, thus forming an object.

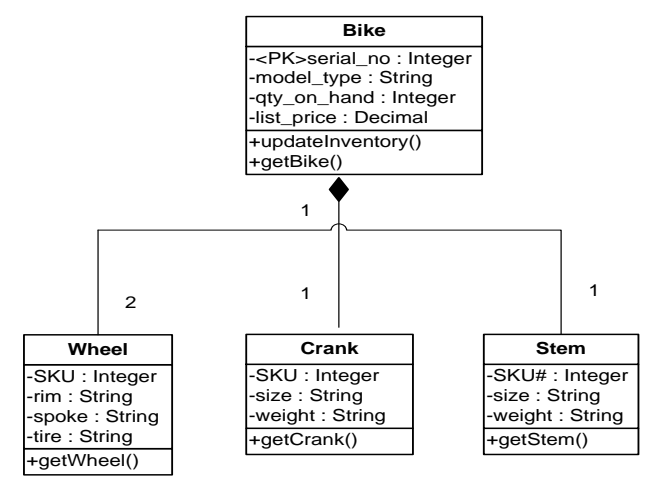

CREATE TYPE wheel_type AS OBJECT(

SKU VARCHAR2(15),

rim VARCHAR2(30),

spoke VARCHAR2(30),

tire VARCHAR2(30));

CREATE TYPE crank_type AS OBJECT

(SKU VARCHAR2(15),

crank_size VARCHAR2(15),

crank_weight VARCHAR2(15));

CREATE TYPE stem_type AS OBJECT(

SKU VARCHAR2(15),

stem_size VARCHAR2(15),

stem_weight VARCHAR2(15)

)

Volume XI, No. 1, 2010

94
The following statement creates nested table types: wheel_type, crank_type and stem_type:

CREATE TYPE nested_table_wheel_type AS

TABLE OF wheel_type;

CREATE TYPE nested_table_crank_type AS

TABLE OF crank_type;

CREATE TYPE nested_table_stem_type AS TABLE OF stem_type;

The following example creates the table named Bike with that contains four nested tables:

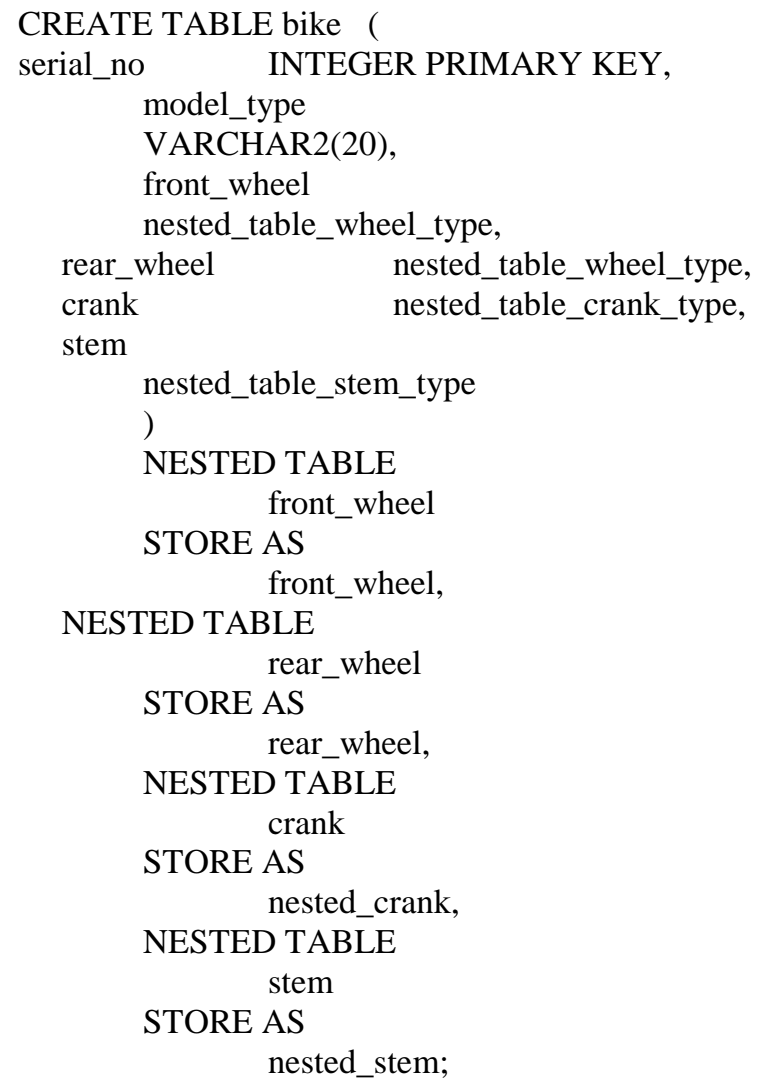

Finally the next statement inserts a row into the Bike table with nested tables using the three defined constructors:

INSERT INTO bike VALUES (1000, 'K2 2.0 Road', nested_table_wheel_type( 
'700x26c' )),

wheel_type('w7023', '4R500', '32 spokes',

nested_table_wheel_type(

wheel_type('w7023', '4R500', '32 spokes',

'700x26c' )),

nested_table_crank_type(

crank_type('c7023', '30X42X52', '4

pounds')),

$$
\begin{aligned}
& \text { nested_table_stem_type( } \\
& \text { stem_type('s7023', 'M5254', '2 pounds'))); }
\end{aligned}
$$

The above example shows that using the NESTED TABLE can implement the composition association, store multiple parts and also speed up the data retrieval speed for the Bike table. The following statement shows the nested tables in the table Bike.

\section{SELECT * from bike;}

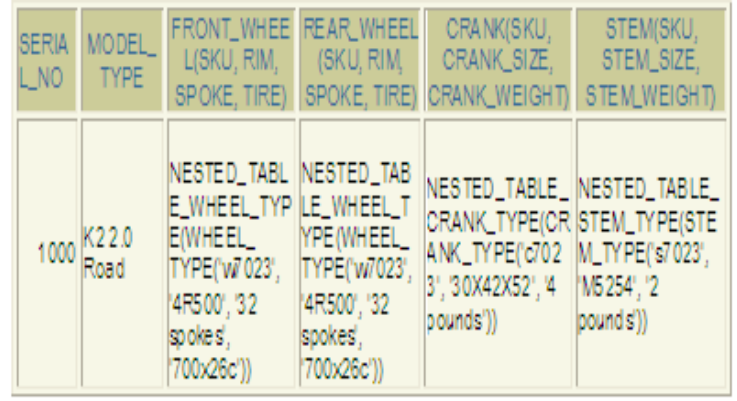

\section{ORDBMS FOR OBJECT INTEGRATION}

The beauty of ORDBMSs is reusability and sharing. Reusability mainly comes from storing data and methods together in object types and performing their functionality on the ORDBMS server, rather than have the methods coded separately in each application. Sharing comes from using user-defined standard data types to make the database structure more standardized [1].

\section{Object Views on a Relational Table}

Object views are virtual object tables, which allow database developers to add OOP structures on top of their existing relational tables and enable them to develop OOP features with existing relational data. The object view is a bridge between the relational database and OOP. Object view creates a layer on top of the relational database so that the database can be viewed in terms of objects [11]. This enables you to develop OOP features with existing relational data. The following statements show how to create the SalesOrder table:

CREATE TABLE SalesOrder ( ord_id NUMBER(10), ord_date DATE, cust_id NUMBER(10), emp_id NUMBER(10));

INSERT INTO SalesOrder VALUES (100,'5-Sep-05', 1, '1000');

INSERT INTO salesOrder VALUES (101, '1-Sep-05', 1, '1000');

The following statements show how to create an object view on the top of the SalesOrder relational table:

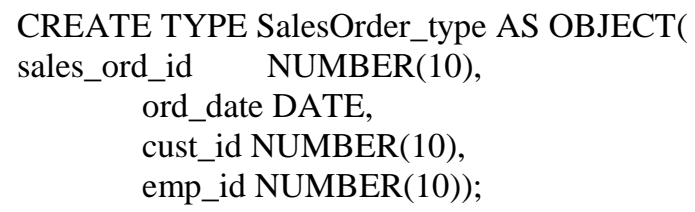

CREATE VIEW customer_order_view OF SalesOrder_type

WITH OBJECT IDENTIFIER
(sales_ord_id) AS

$$
\text { SELECT o.ord_id, o.ord_date, }
$$
o.cust_id, o.emp_id

FROM salesOrder o WHERE o.cust_id = 1;

The following SQL statement generates the view output:

SELECT * FROM customer_order_view;

\begin{tabular}{|c|c|c|c|}
\hline SALES_ORD_ID & ORD_DA TE & CUST_ID & EMP_ID \\
\hline 100 & $05-S E P-05$ & 1 & 1000 \\
\hline 101 & $01-S E P-05$ & 1 & 1000 \\
\hline
\end{tabular}

The object view is a bridge that can be used to create object-oriented applications without modifying existing relational database schemas. By calling object views, relational data can be retrieved, updated, inserted, and deleted as if such data were stored as objects. The following statement can retrieve Analysts as object data from the relational SalesOrder table. Using object views to group 
logically-related data can lead to better database performance.

\section{Inheritance for Object Reuse}

The main advantages of extending the relational data model come from reuse and sharing. If multiple applications use the same set of database objects, then you have created a de facto standard for the database objects, and these objects can be extended [15]. ORDBMSs allow users to define hierarchies of data types. With this feature, users can build subtypes in hierarchies of database types. If users create standard data types to use for all employees, then all of the employees in the database will use the same internal format. Users might want to define a full time employee object type and have that type inherit existing attributes from employee_ty. The full_time_ty type can extend employee_ty with attributes to store the full time employee's salary. The part_time_ty type can extend employee_ty with attributes to store the part-time employee's hourly rates and wages. Inheritance allows for the reuse of the employee_ty object data type. The details are illustrated in the following class diagram:

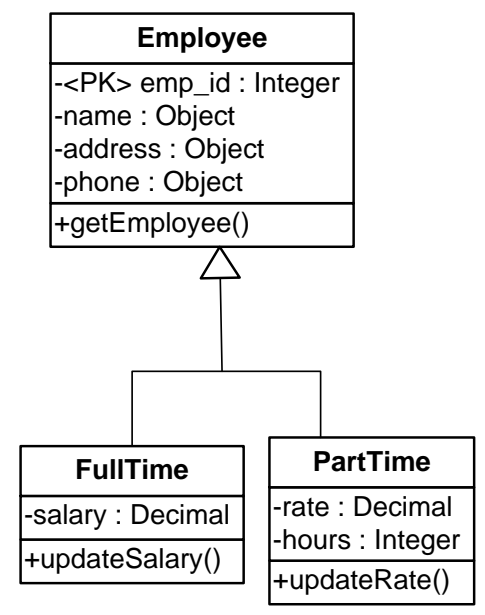

Object type inheritance is one of new features of Oracle 9i. For employee_ty to be inherited from, it must be defined using the NOT FINAL clause because the default is FINAL, meaning that object type cannot be inherited. Oracle 9i can also mark an object type as NOT INSTANTIABLE; this prevents objects of that type derived. Users can mark an object type as NOT INSTANTIABLE when they use the type only as part of another type or as a super_type with NOT FINAL. The following example marks address type as NOT INSTANTIABLE:

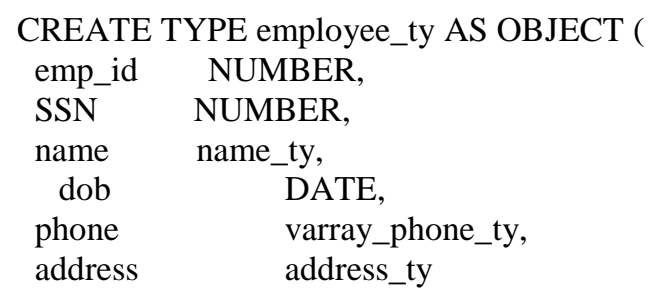

) NOT FINAL NOT INSTANTIABLE;

To define a new subtype full_time_ty inheriting attributes and methods from existing types, users need to use the UNDER clause. Users can then use full_time_ty to define column objects or table objects. For example, the following statement creates an object table named FullTimeEmp.

CREATE TYPE full_time_ty UNDER employee_ty ( Salary NUMBER(8,2));

CREATE TABLE FullTimeEmp of full_time_ty;

The preceding statement creates full_time_typ as a subtype of employee_typ. As a subtype of employee_ty, full_time_ty inherits all the attributes declared in employee_ty and any methods declared in employee_ty. The statement that defines full_time_ty specializes employee_ty by adding a new attribute "salary". New attributes declared in a subtype must have names that are different from the names of any attributes or methods declared in any of its supertypes, higher up in its type hierarchy. The following example inserts row into the FullTimeEmp table. Notice that the additional salary attribute is supplied

INSERT INTO FullTimeEmp VALUES

(1000, 123456789, name_ty('Jim', 'Fox', 'K'), '12MAY-1960',

varray_phone_ty('(626)123-5678', '(323)343-2983', '(626)789-1234'),

Address_ty ('3 Lost Spring Way', 'Orlando', 'FL', 32145), 45000.00);

SELECT * FROM FullTimeEmp; 


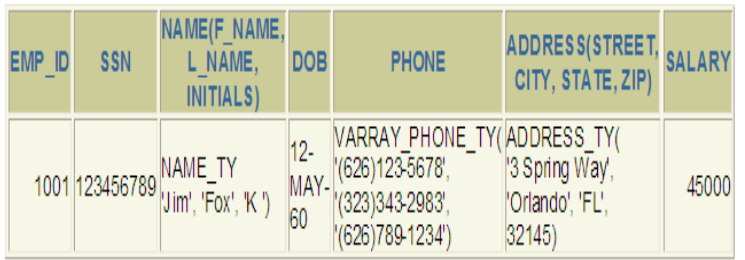

A supertype can have multiple child subtypes called siblings, and these can also have subtypes. The following statement creates another subtype part_time_ty under Employee_ty.

CREATE OR REPLACE TYPE part_time_ty UNDER employee_ty (

rate Number(7,2),

hours Number(3))NOT FINAL;

CREATE TABLE PartTimeEmp of part_time_ty;

A subtype can be defined under another subtype. Again, the new subtype inherits all the 97attributes and methods that its parent type has, both declared and inherited. For example, the following statement defines a new subtype student_part_time _ty under part_time_ty. The new subtype inherits all the attributes and methods of student_part_time _ty and adds two attributes.

CREATE TYPE student_part_time_ty UNDER part_time_ty

(school VARCHAR2(20), year VARCHAR2(10));

\section{Object Integration with Interface}

ORDBMS combines attributes and methods together in the structure of object type. The object type interface includes both attributes and its methods. The public interface declares the data structure and the method header shows how to access the data. This public interface serves as an interface to applications. The private implementation fully defines the specified methods.

Public Interface Specification:

Attribute declarations

Method specifications

Private Implementation

\begin{tabular}{|c|}
\hline Body: \\
\hline Method implementations \\
\hline
\end{tabular}

The following statement displays the public interface of the object type name_type. The output of the name_type public interface shows attributes and method headers as follows:

DESC name_ty;
\begin{tabular}{|l|l|}
\hline Name & Type \\
\hline F_NAME & VARCHAR2(25) \\
\hline L_NAME & VARCHAR2(25) \\
\hline INITIALS & CHAR $(2)$ \\
\hline
\end{tabular}

\section{METHOD RETURNS VARCHAR2 \\ MEMBER FUNCTION FULL_NAME}

Although the user-defined methods are defined with object data within the object type, they can be shared and reused in multiple database application programs. This can result in improved operational efficiency for the IT department, as well, by improving communication and cooperation between applications. An object-relational database schema consists of a number of related tables that forms connected user-defined object-types. Object-types possess all the properties of a class, data abstraction, encapsulation, inheritance and polymorphism. These traits of object-types are embedded in the relational nature of the database; data model, security, concurrency, normalization. In more precise words, the underlying ORDB data model is relational because object data is stored in tables or columns.

\section{CONCLUSION}

The contribution of the paper is that it uniquely provides guidelines on how to use ORDBMS to overcome relational database existing problems and improve database performance in the database development using ORDBMS features. There is some research that has been done in ORDBMS technology as ORDBMSs have become commonplace in recent years. He and Darmont [8] propose the Dynamic Evaluation Framework (DEF) that simulates access pattern changes using configurable styles of change. Pardede, Rahayu, \& Taniar [13] [16] propose an innovative methodology to store XML data into new ORDB data structures, such as user-defined type, row 
type and collection type. The methodology has preserved the conceptual relationship structure in the XML data, including aggregation, composition and association. Mok [12] and Cho, et. al. [2] present a methodology for designing proper nesting structures of user-defined types in object-relational database. The proposed schema trees schema are transformed to Oracle $10 \mathrm{~g}$. Their purpose is to develop an automatic ORDB design tool. Philippi [14] conducts research on the automatic generation of objectrelational mappings into order to overcome objectrelational impedance-mismatch.

So far very little research has been done in using ORDBMS to overcome relational database weaknesses and solve some existing normalization problems. This paper provides the guidelines for the traditional relational database practitioners to solve existing problems using ORDB technology. Many traditional database practitioners consider the ORDBMS technology as complex results in the loss of the essential simplicity and purity of the relational database model and stay away from it. There is a need to provide these professionals with the guidelines for their specific use for their future database development. This paper presents the script templates for them to implement ORDB technology in their career.

The significance of the paper is to promote the utilization of ORDBMS features for problem solving and object reuse and integration among database practitioners. The use of ORDBMS to develop applications can enforce the reuse of varying userdefined object types, provide developers' an integrated view of data and allow multiple database applications to operate cooperatively. Ultimately, this can result in improved operational efficiency for the IT department, increase programmers' productivity, lower development effort, decrease maintenance cost, reduce the defect rate, and raise the applications' reliability. If multiple database applications use the same set of database objects in ORDBMS, a de facto standard for the database objects is created, and these objects can be extended, reused and integrated in the ORDB.

\section{REFERRNCES}

1. Begg, C., \& Connolly, T. (2010). Database systems: A practical approach to design, implementation, and management, 5th Ed. Addison Wesley.

2. Cho, W., Hong, K. \& Loh, W. (2007). Estimating nested selectivity in object-oriented and object-relational databases Information and Software Technology, (49)7, 806-816

3. Connolly, T. and Begg, C. (2006). Database systems: A practical approach to design, implementation, and management, 4th Ed. Addison Wesley.

4. Elmasri, R. \& Navathe, S. (2011). Fundamentals of Database Systems, 6th Edition, Addison Wesley.

5. Fortier, P. (1999). SQL3: Implementing the Object-Relational Database, Osborne McGrawHill,

6. Frank, M. (1995). Object-relational Hybrids, DBMS, 8/8, 46-56.

7. Garcia-Molina, H., Ullman, J. \& Widom, J. 2003. Database Systems: The Complete Book, Prentice Hall, Upper Saddle River.

8. He, Z., \& Jérôme, D. (2005). Evaluating the Dynamic Behavior of Database Applications, Journal of Database Management; 16:2, 21-45.

9. Hoffer, J., Prescott, M., \& Topi, H., 2009 Modern Database Management, 9th Edition, Pearson Prentice Hall.

10. Krishnamurthy, Banerjee and Nori, 1999. Bringing object-relational technology to the mainstream, Proceedings of the ACM SIGMOD International Conference on Management of Data and Symposium on Principles of Database Systems, Philadelphia, PA

11. Loney, K. \& Koch, G. (2002) Oracle 9i: The complete reference, Oracle Press/McGrawHill/Osborne. 
12. Mok, W. Y. (2007) Designing nesting structures of user-defined types in object-relational databases, Information and Software Technology 49, 1017-1029.

13. Pardede, E., Rahayu, J. Wenny, T. \& Taniar, D., 2006, Object-relational complex structures for XML, Information \& Software Technology, 48(6), 370-384.

14. Philippi, S. 2004, Model driven generation and testing of object-relational mappings, Journal of Systems and Software, 77:2, 193-207.
15. Price, J. 2002. Oracle9i, JDBC Programming, Oracle Press/McGraw-Hill/Osborne

16. Rahayu, J. W., Taniar, D. And Pardede, E. (2005) Object-Oriented Oracle, IRM Press

17. Silberschatz, A., Korth, H. and Sudarshan, S. 2009, Database System Concepts, six Edition, McGraw-Hill

18. Stonebraker M. and Moore, D. 1996. Objectrelational DBMSs: the Next Great Wave. San Francisco, CA: Morgan Kaufmann Publishers, Inc. 